POLIGRAFÍAS. REVISTA DE TEORÍA LITERARIA Y LITERATURA COMPARADA. NÚMERO 1. NUEVA ÉPOCA.

( ) UNAM TODOS LOS DERECHOS RESERVADOS

https://doi.org/10.22201/ffyl.poligrafiasnuevaepoca.2011.1.1655

\title{
El descubrimiento científico: las dos caras de Jano
}

NOÉ ZÚÑIGA VILLARREAL Instituto de Química, UNAM

Jorge Volpi, mexicano, miembro del llamado "grupo del crack" (Poniatowska) junto con escritores como Ignacio Padilla, Eloy Urroz y Ricardo Chávez, entre otros, "revive" en 1999 el Premio Biblioteca Breve de Seix Barral (que había sido suspendido desde 1972) con la novela En Busca de Klingsor. La trama gira alrededor del poder: el poder de la ciencia o, mejor dicho, el poder que ejercen los que manipulan la ciencia y el mal (visto como una causa de perjuicio para la humanidad). Obra ambientada en la Alemania de posguerra (de la Segunda Guerra Mundial), En busca de Klingsor registra las andanzas de un joven físico norteamericano, Francis P. Bacon, tras un personaje, Klingsor (este nombre aparece en el Cuento del Grial de Chrétien de Troyes y en el Perceval de Gottfried von Eschenbach; el personaje fue popularizado por Richard Wagner en su ópera Parsifal), que, según la novela, era el nombre en clave del asesor científico del Führer. La narración comienza con el testimonio de Gustav Links, matemático que "participó" en el atentado del 20 de julio de 1944 contra Hitler. Links es el narrador omnisciente y, en el tiempo de la narración, ya ha estado recluido por mucho tiempo en un hospital psiquiátrico (reminiscencias de Oskar Matzerath en El tambor de hojalata de Günter Grass). La credibilidad de la narración, entonces, se pone en duda, pues un personaje que funja como narrador omnisciente necesariamente tendrá que relatar algunos acontecimientos recurriendo a su imaginación. Volpi, en un intento por justificar lo anterior, comenta a través de Gustav Links:

No me es difícil suponer que, después de la historia que he expuesto sobre el teniente Francis $P$. Bacon, una incómoda pregunta queda en el aire: si este señor Gustav Links, matemático de la Universidad de Leipzig, ha insistido en decir que es el narrador de los hechos ¿cómo es posible que conozca hasta los detalles más inconcebibles sobre otra persona, esto es, sobre el teniente Bacon?

La inquietud me parece legítima y por eso me he tomado la libertad de incluir este apartado. Se trata de una duda razonable, porque de su resolución depende la credibilidad de mi 
relato. Un hombre de ciencia como yo, sabe que, sin pruebas, cualquier teoría se desmorona en el aire. He aquí mi réplica: no puedo decir que todos los hechos que he descrito sean verdaderos -de ahí que los haya denominado hipótesis-, pues lo cierto es que no me tocó presenciarlos. ¿Entonces qué puedo alegar a mi favor? Algo muy sencillo: el propio teniente Francis P. Bacon se encargó de hablarme de su pasado durante las largas horas que pasamos juntos (Volpi, 1999: 111).

El personaje narrador comenta lo anterior después que ha descrito en la novela la niñez y juventud de Francis P. Bacon. En este punto se pueden visualizar dos componentes de incertidumbre: en la primera se involucra a un personaje cuya narración es poco confiable pues, aparte de los años que hay de por medio entre los hechos que narra y la propia narración, es un paciente en un hospital psiquiátrico; el segundo componente involucra a este mismo paciente fungiendo ahora como narrador omnisciente que, como tal, es capaz de tener acceso a los pensamientos más íntimos de los personajes y hacérselos saber al lector. Si se toma en cuenta el primer componente, se introduce un alto grado de incertidumbre; los acontecimientos, entonces, están sumergidos en una atmósfera cargada de sospechas causadas por el narrador que demandan del lector, una vez que ha llegado al final de la novela, una cuidadosa revaloración de los hechos, sin que esto garantice una mayor aprehensión de la trama, pues el narrador no es confiable. Si a lo anterior se adiciona el segundo componente de incertidumbre, como lo hace Volpi, entonces se puede llegar al extremo de considerar que toda la novela es una mentira; pero esto no es lo peor (pues toda obra de ficción es mentira), sino que se pone en duda la verosimilitud, no ya del personaje, sino de la novela como tal.

Hablar del conocimiento y el poder que éste conlleva no es tarea fácil; sobre todo si se trata del conocimiento científico. ${ }^{1}$ El cambio que la ciencia ha operado a lo largo del siglo pasado ha sido espectacular: se puede afirmar que la mayor influencia de la ciencia y la tecnología sobre la vida cotidiana de los seres humanos, a lo largo de su estancia sobre la tierra, ha sido experimentada en el siglo XX. Esto no quiere decir que no se hayan hecho grandes descubrimientos científicos a lo largo de la historia del ser humano, sino que la aplicación y repercusión de los descubrimientos científicos en la sociedad antes del siglo XX fueron más lentas, como en el caso de la observación de los astros, un área íntimamente ligada al avance de la sociedad al poner de manifiesto que la tierra no era el centro del universo, como alguna vez

\footnotetext{
${ }^{1}$ Ver, por ejemplo, Eduardo Nicol (1997): Los principios de la ciencia.
} 
se había pensado, ocasionando fuertes controversias entre las ideologías establecidas y la observación experimental defendida por Copérnico, Galileo o Giordano Bruno² (quien murió en la hoguera por defender sus puntos de vista); la asimilación de estas observaciones por parte de la sociedad tardó varios siglos; mientras que, a partir del siglo pasado, un proceso semejante tardaría algunos años. El revuelo que las ideas de los descubrimientos causa en su momento suele ser mayúsculo, e, inclusive, nocivo para quienes los proponen; una vez que tal idea ya ha sido aceptada, pasa a formar parte del corpus cultural de la humanidad.

El avance de la ciencia en el siglo $X X,^{3}$ entonces, ha sido tan acelerado que los valores éticos han tenido que sufrir cambios de acuerdo con los nuevos descubrimientos, como la clonación o la producción de alimentos manipulados genéticamente. El poder, entendido como forma de manipulación social, no ha escapado de los avatares del desarrollo de la ciencia; de aquí que ésta, con su ingente papel en la sociedad contemporánea, haya sido utilizada como un instrumento de poder.

El precio que la humanidad ha pagado por el conocimiento ya ha sido ponderado mucho antes de la era moderna. Fausto en The Tragical History of Doctor Faustus de Christopher Marlowe, basado en una leyenda medieval (que después retomarán muchos escritores), vende su alma al diablo con tal de acceder al conocimiento científico y nos deja una idea muy clara de lo que el hombre es capaz por tratar de comprender los mecanismos internos que rigen la naturaleza. En la tradición judeo-cristiana, el simple deseo por el conocimiento era visto como peligroso y maligno: el mito edénico asociaba la búsqueda del conocimiento con la desobediencia a Dios, con el orgullo y la soberbia y, en última instancia, con la caída del hombre. El conocimiento científico, por lo tanto, ha pasado por varias etapas que corresponden a las diferentes concepciones de la imagen del científico. El objetivo de este artículo es, pues, explorar el papel de los científicos en la sociedad, tomando como punto de partida la experiencia alemana consignada en la novela de Jorge Volpi, En busca de Klingsor, con el fin de puntualizar que el descubrimiento científico puesto en manos inadecuadas puede resultar no sólo pernicioso para el avance de la ciencia, sino destructivo para la humanidad.

${ }^{2}$ Cfr. John Gribbin (2003): Historia de la Ciencia, 1543-2001.

${ }^{3}$ Ver, por ejemplo, John D. Bernal (1986): La ciencia en la historia. 
El entorno social del científico alemán en la obra de Volpi corresponde al de la primera mitad del siglo XX; como lo atestigua Gustav Links, narrador y a la vez personaje; matemático alemán, quien antes de embarcarse, junto con el teniente Francis P. Bacon en su búsqueda para descubrir la identidad de Klingsor, declara:

Cuando nací, el mundo era un sitio ordenado, un cosmos serio y meticuloso en el cual los errores -las guerras, el dolor, el miedo- no eran más que lamentables excepciones debidas a la impericia. Mis padres, y los padres de mis padres, creían que la humanidad progresaba linealmente, desde el horror de la edad de las cavernas, hasta la brillantez del futuro, como si la historia no fuese más que un cable tendido entre dos postes de luz o, para utilizar la metáfora que mejor define al siglo XIX, como una vía férrea que une, al fin, dos poblados remotos. En medio de este escenario, nacer era poco más que un trámite. A partir de ahí, la severa educación que se nos impartía bastaba para modelarnos, para hacernos hombres de bien y para asegurar nuestro porvenir... Los valores que se nos enseñaban entonces eran muy simples: disciplina, austeridad, nacionalismo. ¡Esta empresa parecía tan hermosa y, a la vez, tan simple! Si la regla del mundo era el progreso, las existencias individuales debían plegarse al mismo esquema. ¿Por qué algo habría de fallar? Si se planeaba con suficiente cuidado la formación de un niño, si se le proporcionaban las herramientas que asegurasen su desarrollo, su crecimiento físico y espiritual, y si se forjaba su carácter como si fuese, en efecto, una lámina de bronce sobre el yunque de la moral, poco a poco la sociedad podría deshacerse de los locos, los criminales y los mendigos, asegurándose una comunidad de hombres honrados, ricos, alegres y piadosos (Volpi, 1999: 114).

Las características que Volpi resalta en este personaje se derivan del momento histórico del científico. El orden en la cultura germana era un valor que debía cultivarse a toda costa para trascender en todos los ámbitos de la vida. Esto, se suponía, garantizaba la estabilidad de las personas, dándoles un sentimiento de seguridad; se movían en un mundo ordenado donde había poco lugar para el caos, creían ellos, lo cual, a su vez, tendría consecuencias "benignas" para la sociedad; estos mismos "valores" son los que se retomarían en el período nacional socialista con Hitler. La derrota de Gustav Links como ser humano se evidencia al pertenecer al grupo de indeseables sociales: locos, criminales y mendigos, ya que, al momento de hacer estas reminiscencias, ya ha estado recluido en una institución mental por un largo periodo. Estamos ante un científico que es producto de la sociedad en la que se desarrolló, la cual es descrita minuciosamente, revelando la escrupulosa investigación realizada por el escritor. La noción de que el científico y sus investigaciones son producto de su entorno social no es tan obvia como pudiera parecer a primera vista; ya que por mucho tiempo se consideró que el conocimiento 
científico era absoluto y que se regía por métodos rígidos muy independientes del entorno social donde se gestaba. ${ }^{4}$

Otro personaje de la novela, Francis P. Bacon, joven militar cuya tarea es descubrir la personalidad de Klingsor, es un físico norteamericano; las razones que lo conducirían a la ciencia se describen así:

En el colegio, el rechazo hacia sus semejantes se fue diluyendo lentamente gracias a su creciente admiración por las leyes naturales (lo cual incluía, al menos en principio, cierta admiración por la humanidad). Aunque no todo lo que ocurría podía ser explicado por la razón, al menos la ciencia le aseguraba un camino recto hacia el conocimiento. Lo más importante era que, al averiguar qué leyes regían el mundo, podría llegar a tener algún control sobre los demás. Sin desprenderse de su desconfianza original, Frank la arrinconó en una parte de su memoria a la cual recurría cada vez con menos frecuencia.

Una mañana se levantó con un humor franco y expansivo; sin saber exactamente la razón, había decidido alejarse de las matemáticas puras, con su red de abstracciones y fórmulas incognoscibles, para acercarse al terreno más sólido y concreto de la física. [...] Su tarea, ahora, no sería combinar números como un esquizofrénico revuelve palabras, sino adentrarse en los componentes básicos del universo: la materia, la luz, la energía. [...] Bacon se entusiasmó con la rama más novedosa, frágil e impráctica de la física: el estudio de los átomos y la muy reciente teoría cuántica. Ahí, otra vez, no había nada tangible: los nombres de los objetos que analizaba electrones, fuerzas, campos magnéticos- eran las etiquetas de unos entes tan bizarros como los números (Volpi, 1999: 48-49).

Bacon encuentra su camino a la ciencia por motivaciones un tanto idealistas, es decir, su eje rector es la adquisición del conocimiento per se; no obstante, la concepción idealista se va diluyendo para dar paso a un objetivo más básico: el control sobre los demás. Esta ideología no es inocente, y refleja muy bien el medio social en el que Bacon ha estado sumergido. La ambición por manipular a los demás permea las esferas políticas llegando, incluso, a la disputa del poder entre naciones. Es aquí donde la ciencia sirve como medio para obtener intereses que están muy alejados de ella. Los descubrimientos científicos no tienen que ver con la moralidad, aunque el científico, como miembro de la sociedad, sí. La ciencia, como generadora de poder, tiene que ser vista, ahora, como una empresa política, socialmente organizada. Muchos descubrimientos científicos han sido utilizados en aras del bien y del mal (beneficio o destrucción de la humanidad); sin embargo, el propio descubridor, poco tenía que decir al

\footnotetext{
${ }^{4}$ Cfr. Ana Rosa Pérez Ransanz (1999): Kuhn y el cambio científico.
} 
respecto: la finalidad de sus investigaciones eran dictadas por intereses ajenos a la ciencia. Volpi muestra, en Bacon, un científico poco comprometido con su entorno social, aunque es totalmente un reflejo de éste. La carrera de Bacon, joven científico prometedor, se ve truncada por problemas personales que lo conducirán a la búsqueda de Klingsor; el escritor de esta forma desmitifica la imagen del científico, pues a la larga las pasiones de Bacon triunfarán sobre su raciocinio.

El desarrollo de la mecánica cuántica y una de sus consecuencias prácticas, la bomba atómica, enmarcadas en una Alemania exhausta por la guerra, junto con la exploración de las relaciones personales de unos cuantos seres, cuyas pasiones los manipularán al final de la novela, son temas que acomete Volpi en En busca de Klingsor, el tema científico lo presenta como una tragedia: a través del manido binomio: maldad y búsqueda del conocimiento (pero ahora con fines bélicos). Sin embargo, el héroe trágico no es una persona en particular, sino más bien un grupo formado por políticos y científicos con pretensiones políticas. Y la tragedia se extendió de este grupo de poder a la sociedad civil. Las tragedias personales, por su lado, un tanto despegadas del ámbito científico (de hecho en algún momento parece que se están leyendo dos novelas con temas independientes), tienen al amor como medio y objetivo.

La novela de Volpi describe, entonces, una sociedad gris, devastada por la guerra; también hurga en el pasado inmediato: antecedentes socio-políticos que dieron lugar al Tercer Reich; no obstante, la falta de documentación científica es conspicua y, para el lector no especializado, puede suponer una falsa impresión respecto a la ciencia alemana bajo el régimen nacional socialista. La época de oro guillermina (de 1870 a 1914) propició que la ciencia y la tecnología en Alemania florecieran aceleradamente. El káiser Wilhelm II hizo posible la creación de la Sociedad Kaiser-Wilhelm, la cual dio lugar a la Sociedad Max-Planck, ambas con sus institutos de investigación correspondientes. Es así que a finales de la primera década del siglo XX, Alemania había llegado a ser la meca internacional de la ciencia. Los científicos de varias partes del mundo, que se dedicaban tanto a la investigación básica como aplicada, acudían a las universidades alemanas para participar en las conferencias y seminarios que ahí se ofrecían; el idioma alemán se constituyó en la lingua franca entre los hombres de ciencia. (De los 100 premios Nóbel de ciencia concedidos entre 1901 y 1932, había más de 30 otorgados a científicos 
alemanes). La deuda de Europa y Norteamérica a la influencia alemana (antes del impacto de Hitler, Goebbels y Himmler) ha sido enorme, pero también compleja y ambivalente. El término alemán para ciencia, Wissenschaft, incorporó tradicionalmente un vasto conjunto de disciplinas intelectuales donde los germanoparlantes, incluyendo a los austriacos, destacaron, dejando profunda influencia en el pensamiento occidental.

Cuando Hitler llegó al poder en 1933, la ciencia, la medicina y la tecnología fueron puestas al servicio del nuevo régimen. El Tercer Reich necesitaba un espíritu de cooperación: todos los recursos tendrían que ser encauzados hacia un mismo fin. La educación, los medios, la psicología y las comunicaciones se tuvieron que adaptar a la ideología nacional-socialista con el objeto de canalizar y dar forma a la opinión pública en este cambio político. Los científicos, con pocas excepciones, cedieron a las presiones impuestas sobre ellos. Como comenta el historiador Joseph Haberer: "the scientific leadership engaged in 'expediency and compliance' and colluded with victimization of members of the community" (Cornwell, 2003: 8); lo que es más, algunos grupos, entre los que destacaron los médicos y los antropólogos, no sólo consintieron, sino que tomaron papeles protagónicos promoviendo políticas racistas; en algunos casos, un segmento de la comunidad científica oprimía y coaccionaba al otro: la física experimental, por citar un ejemplo, bajo la influencia de algunos científicos nazis, era reconocida como auténtica, mientras, la física teórica era considerada como una "maquinación" judía; como irónica y premonitoriamente lo había manifestado Albert Einstein en la Sorbona en 1920: “Si mi teoría de la relatividad resulta correcta, Alemania me acogerá como un alemán, y Francia me declarará ciudadano del mundo; si mi teoría resulta falsa, Francia proclamará que soy alemán y Alemania declarará que soy judío" (Gratzer, 2000: 17). En este ambiente de represión, superioridad étnica e intransigencia bélica, se desarrollaron muchos científicos alemanes sin que esto supusiera problemas para sus carreras científicas. Esta complicidad entre la política y los científicos no era nueva, ya en la Primera Guerra Mundial el repudio internacional que suscitó la invasión de Francia y Bélgica por Alemania fue combatido por la opinión pública alemana, representada por varios científicos prominentes, tanto en aquel entonces como en el régimen nacional socialista, a través de una protesta intitulada "llamado al mundo de la cultura" (Aufruf an die Kulturwelt) el 4 de octubre de 1914 y que apareció en los principales periódicos alemanes de la 
época. La abstención de Einstein a firmar tal documento atrajo fuertes críticas y repudio contra el científico alemán. A este incipiente repudio siguieron ataques en el terreno profesional: en 1920, en el Congreso de médicos e investigadores en ciencias naturales (Tagung der Naturforscher und Ärzte), llevado a cabo en Bad, Nauheim, Philipp Lenard arremete contra la teoría de la relatividad, lo que más tarde desembocaría en el artículo que Johannes Stark (Premio Nóbel de física en 1919) envió a la revista británica Nature titulado “The Pragmatic and the Dogmatic Spirit in Physics", donde establecería la ominosa y espuria distinción entre física aria y física judía. No obstante, en el régimen nazi, no fue la física la única ciencia que se cultivaría en Alemania; al respecto, Volpi hace patente en En busca de Klingsor que Klingsor, el personaje, tendría que ser alguien relacionado con la física cuántica, no precisamente un físico, quizá un matemático, dejando así de lado una parte importante de los médicos y científicos naturales que realizaron significativos avances en el ámbito científico. Los químicos y los bioquímicos en el Tercer Reich también sufrieron los avatares del régimen nazi y, a la par que los tan estudiados físicos, también los químicos y los bioquímicos (en adelante (bio)químicos para denotar ambas disciplinas) respondieron con gestos sublimes, algunos, y con asimilación destructiva del poder, otros. Dicho de otra forma, Klingsor bien hubiera podido ser un (bio)químico, con un afán inconmensurable por agradar al nuevo orden político, deseoso de servir a Hitler sin importar las consecuencias.

Es bien sabido que un resultado, execrable por lo demás, de la liza entre alemanes y aliados fue la creación de una de las armas de destrucción masiva más perniciosa para la humanidad: la bomba atómica, aunque no fueron los alemanes, precisamente, quienes la probaron sobre la población civil; sin embargo, la necesidad por encontrar un arma capaz de acabar con el enemigo ha sido un lugar común en las guerras. Y esto no fue la excepción en la Primera Guerra Mundial; contraviniendo flagrantemente la Convención de la Haya, el 22 de abril de 1915 en la línea alemana asentada en Ypres, Francia, se utilizó cloro contra el frente francés algeriano: se ocuparon 5,730 cilindros cada uno con un peso de 200 libras con consecuencias desastrosas para el frente francés. Y bien, ¿cómo nació la idea de usar este tipo de armas contra el enemigo? ¿Fue un político? No. Fue un científico: Fritz Haber, específicamente, un químico judío-alemán. La trayectoria científica de Haber es el ejemplo paradigmático de las 
consecuencias del uso del poder a través de la ciencia, para bien y para mal. Este químico desarrolló, además de sus extensivos estudios sobre gases de guerra, el proceso Haber-Bosch, junto con Carl Bosch, que consiste en la síntesis de amoníaco, compuesto profusamente utilizado en la manufactura de fertilizantes y explosivos, a partir de nitrógeno e hidrógeno gaseosos, utilizando un catalizador (sustancia que acelera la velocidad de reacción); este proceso se conoce como "fijación de nitrógeno" y le valió a Haber tanto el premio Nóbel de química en 1918, como gran reconocimiento en el medio académico alemán de aquel entonces.

Los gases que se utilizaron en la guerra mundial, como bien se sabe, tienen efectos devastadores para los seres vivos. El escritor Erich Maria Remarque en su novela Im Westen nichts Neues (Sin novedad en el frente) describe:

Einen Unterstand voll finden wir mit blauen Köpfen und schwarzen Lippen. In einem Trichter haben sie die Masken zu früh losgemacht. Sie wußten nicht, daß sich das Gas auf dem Grunde am längsten hält; als sie andere ohne Maske oben sahen, rissen sie sie auch ab und schluckten noch genug, um sich die Lungen zu verbrennen. Ihr Zustand ist hoffnunglos, sie würgen sich mit Blutstürzen und Erstickungsanfällen zu Tode. ${ }^{5}$

Entre las sustancias químicas que Haber desarrolló como arma letal de lesa humanidad se encuentra el ácido hidrociánico (solución acuosa del ácido cianhídrico), que más tarde se conocería con el nombre de Zyklon $B$ y que serviría para aniquilar a muchos prisioneros de campos de concentración alemanes donde, irónicamente, fenecieron muchos conocidos y parientes de Fritz Haber: un hombre famoso, reconocido y sediento de poder, aunque con una gran desventaja: sus orígenes judíos. Un personaje de esta índole bien podría ponerse el traje de Klingsor. Aunque Haber, conocido en el mundo científico como un talentoso e infatigable organizador y promotor de la ciencia, uno de los primeros científicos con más éxito en fraguar un vínculo entre la investigación y la industria, no pudo haber sido tal, pues murió a principios de 1934, cuando estaba en el Instituto Daniel Sieff (más tarde conocido como el Instituto

\footnotetext{
${ }^{5}$ Encontramos un refugio subterráneo lleno de ellos, con caras azuladas y labios negros. Los de dentro de un foso se quitaron las mascarillas antigás demasiado pronto. No sabían que el gas se mantiene más tiempo en los agujeros; cuando vieron que los de arriba iban sin mascarilla, se quitaron la suya y respiraron suficiente gas como para quemarles los pulmones. Su estado es desesperado; las bocanadas de sangre los estrangulaban y unas terribles crisis de ahogo les llevan irremediablemente a la muerte.
} 
Weizmann) en Palestina, después de haber renunciado a su posición académica y abandonado Alemania por voluntad propia.

Hannah Arendt, en su libro Eichmann en Jerusalén (1963), propone la tesis de que la maldad en el Tercer Reich se originó no únicamente por los asesinos fanáticos de las SS, sino también por el hombre promedio, común y corriente que no alza su voz ante los dictadores convirtiéndose en cómplice de sus crímenes. Este punto de vista ha sido analizado y, al mismo tiempo, sostenido por recientes descubrimientos que dan luz sobre el comportamiento de los científicos, médicos e ingenieros en el régimen nacional-socialista; según Deichmann en su artículo "Chemists and Biochemists during the National Socialist Era" (2002), muchos científicos, más que ser obligados a participar, se involucraban libre y dinámicamente. Volpi, en su libro En busca de Klingsor, muestra un grupo de físicos alemanes cuya responsabilidad moral está en tela de juicio: baja del pedestal al científico para situarlo al nivel de cualquier ser humano: gran acierto; sin embargo, las relaciones personales de los científicos desmitificados, sobre todo las amorosas, caen en un terreno farragoso que distan de lo creíble.

La dimensión humana que confiere Volpi a sus físicos denota una cuidadosa investigación de la época desde una perspectiva actual; al respecto Deichmann escribe:

German physicists were unsuccessful in the construction of a nuclear reactor and an atomic bomb. Their representatives, especially Werner Heisenberg and Carl-Friederich von Weizsäcker, spread the rumor after the war that German physicists had not produced the atom bomb for moral reasons. Rober Jungk, who from a lack of background knowledge had contributed considerably to the spread of this rumor in 1956 with his book Heller als Tausend Sonnen, recognized later that he had been misled. Thus in the preface to Die Uranmaschine by Mark Walker he wrote: "That with the translation into many languages of my 1956 book Heller als Tausend Sonnen I had contributed to the myth of passive resistance of the important physicist is attributable to my high esteem for these personalities which I have had since to recognize as inappropriate." This legend was finally destroyed in Germany in 1992 when the British government released tape recordings which documented discussions with German nuclear physicists interned in the British Farm Hall after the war. In 1947 the physicist Samuel Goudsmit had already showed in the USA that this legend had been invented by a number of German physicists themselves, in particular C.-F. von Weizsäcker, in Farm Hall when they received news of the dropping of the American atomic bomb on Hiroshima. Goudsmit attributed the failure of the German uranium project under Werner Heisenberg to deficiencies in scientific organization, the errors and inability of individual scientists and their complacency (2002: 1323). 
La leyenda de la resistencia pasiva, al analizarse bajo una nueva perspectiva, es destruida y renace para dar lugar a una nueva leyenda: la ciencia perjudica y conlleva la destrucción del planeta. Esto no es muy sorprendente, ya que mientras los "malos" no contaban con la tecnología adecuada para fabricar una bomba atómica, los "buenos" tomaban la delantera y la hacían detonar en pequeñas islas destruyendo miles de vidas inocentes; se afianza la personalidad del científico como una suerte de Dr. Jekyll y Mr. Hyde, pero ahora en un escenario cargado de tintes políticos.

Volpi presenta su obra de una forma poco convencional: despega con leyes y corolarios continuando con hipótesis y disquisiciones para aterrizar en diálogos, a manera de un manuscrito científico; una reminiscencia del libro Of the Proficience and Advancement of Learning, Divine and Human de Francis Bacon, mezclado con Los Diálogos de Platón y algún manual de matemáticas. Pero no sólo esto, la técnica epistolar usada por Volpi tiene ecos del escritor irlandés John Banville en su novela Doctor Copernicus, obra en la que las cartas, aparte de poner en comunicación a los personajes, sirven como indicadores de los puestos eclesiásticos que los interlocutores han alcanzado.

El tema de esta novela no es común en la literatura hispanoamericana, ya que cuando se habla de ciencia, se hace de la nacional y cuando se tratan situaciones en el extranjero (o del extranjero) están alejadas de la ciencia. En el presente caso se conjuntan dos vertientes: ciencia de un país extranjero, desde el punto de vista de un mexicano (en realidad no tiene nada que ver la nacionalidad en esta obra, aunque, conscientes de la de Volpi, el mérito es mayor). En busca de Klingsor se alinea con una vena fuertemente policíaca, muy semejante a El nombre de la rosa de Umberto Eco, mutatis mutandi: originales aventuras laberínticas, perfectas para los lectores cultos (la ciencia también es cultura) como para los que se dejan deslumbrar por el placer del entretenimiento.

La imagen del científico que Volpi retoma es la de un romántico: el científico en la soledad de su laboratorio haciendo descubrimientos; sólo que en el caso de Heisenberg (fuerte candidato a ser Klingsor), su laboratorio será su propia cabeza, y en el capítulo "Werner Heisenberg, o de la tristeza", Volpi presenta a Heisenberg frente a la naturaleza como un solitario en actitud de profunda meditación y se impone el recuerdo del verso de Goethe “Über 
allen Gipfeln ist Ruh", (Sobre todas las cumbres está la paz). La imagen de la persona frente a la naturaleza que Volpi describe poéticamente de la página 248 a la 251, cuando Heisenberg llega a la mecánica matricial, parece estar inspirada en una pintura del romántico alemán Caspar David Friederich (1774-1840), El caminante sobre el mar de niebla, (hacia 1818), donde se presenta una imagen dual: por una lado la serenidad del personaje y, por el otro, la voluptuosidad de la naturaleza. Heisenberg, en En busca de Klingsor, se encuentra solo, sentado en un acantilado de la isla de Helgoland, observando el mar al atardecer; se contagia de la furia de los elementos naturales para ponerlos a tono con la tormenta que se está gestando en su mente al saber que sus esfuerzos científicos han fructificado.

Volpi apuesta por un nuevo tipo de novela, abre una veta poco o nunca explorada por escritores de habla hispana. Su éxito editorial fue grande: fue reconocido con el Premio Biblioteca Breve Seix Barral en 1999. Según el escritor, esta novela era la primera de una trilogía cuya segunda parte ya ha sido publicada, El fin de la locura, que, al parecer, no ha gozado de la misma aceptación por parte de la crítica literaria. La escritura de En busca de Klingsor duró cinco años, y esto es un indicador de que la escritura de una novela de tal calibre no es fácil; las investigaciones en el tiempo histórico que consigna Volpi fueron minuciosas y aunque las fuentes son muchas, o quizá por esto, el manejo de la información tuvo que haber sido muy selectivo. De aquí que no se pueda esperar de tal obra un panorama totalizador de la ciencia en Alemania nazi; de lo contrario, la novela correría el peligro de ser un mero volumen de registros político-científicos de la época. Los conceptos científicos que maneja el escritor han tenido que ser forzosamente poco profundos, ya que de lo contrario el libro podría llegar a ser incomprendido; algún crítico ha comentado que, para disfrutar mejor este libro, habría que regresar a los apuntes de ciencia del bachillerato; no creo que éste sea el caso, pues, como ya mencioné, los conceptos científicos son presentados superficialmente y no se necesita un mayor entendimiento de los conceptos científicos que los que la propia obra explica. Novelas como En busca de Klingsor hacen falta en Latinoamérica con el objetivo de llenar la gran carencia de conocimiento científico, a nivel divulgación, y su relación con la sociedad; y ya que, al parecer, los científicos no se ven muy deseosos de involucrarse en la divulgación de la ciencia, es loable que un literato lo haga. El progreso de la humanidad a través de la ciencia no parece que vaya a 
disminuir en el futuro próximo, por el contrario; de aquí que el individuo que se preocupe por su entorno deberá estar enterado acerca de los descubrimientos científicos y su impacto en la sociedad, pues de no hacerlo se corre el peligro de allanar el camino hacia un estado totalitario donde los poseedores del conocimiento serían grupos reducidos de poder. 


\section{Bibliografía}

CORNWELL, John (2003): Hitler's Scientists. EE UU: Penguin.

DEICHMANN, Ute (2002): “Chemists and Biochemists during the National Socialist Era". En Angew. Chem. Int. Ed. Engl. Núm. 41, pp. 1310-1328.

GRATZER, Walter (2000): "The waning of the Golden Age. Politics, war and the decline of German science". En Nature. Núm. 403, pp. 17-19.

PONIATOWSKA, Elena: “Box y literatura del crack”. Disponible en http:search.ntscape.com. [Consulta: 26 de junio de 2003].

STERN, Fritz (2003): El mundo alemán de Einstein. Barcelona: Paidós.

VOLPI, Jorge (1999): En busca de Klingsor. Barcelona: Seix-Barral. 